\title{
Microvascular Function in Viable Myocardium After Chronic Infarction Does Not Influence Fractional Flow Reserve Measurements
}

\author{
Koen M. Marques ${ }^{1,2}$, Paul Knaapen ${ }^{1,2}$, Ronald Boellaard ${ }^{2,3}$, Adriaan A. Lammertsma ${ }^{2,3}$, Nico Westerhof ${ }^{2,4}$, \\ and Frans C. Visser ${ }^{1,2}$ \\ ${ }^{I}$ Department of Cardiology, VU University Medical Center, Amsterdam, The Netherlands; ${ }^{2} I C a R-V U$, VU University Medical Center, \\ Amsterdam, The Netherlands; ${ }^{3}$ Department of Nuclear Medicine and PET Research, VU University Medical Center, Amsterdam, The \\ Netherlands; and ${ }^{4}$ Department of Pulmonary Diseases and Physiology, VU University Medical Center, Amsterdam, The Netherlands
}

Fractional flow reserve (FFR) is an index of coronary stenosis severity. FFR is the ratio of hyperemic myocardial flow in the stenotic area to maximal flow in that same territory without stenosis and can be measured with a pressure wire. In patients with prior infarction, measuring FFR in infarct-related arteries may be different for 2 reasons: a smaller mass of viable myocardium depending on the stenotic infarct-related artery and greater microvascular resistance in the infarcted area than in the reference area. When microvascular resistance does not differ between the infarcted and the reference areas, FFR should equal relative flow reserve (RFR). RFR is the ratio of myocardial blood flow in the stenotic area to blood flow in a normally perfused reference area, at maximal hyperemia. $\mathrm{H}_{2}{ }^{15} \mathrm{O}$ PET measures myocardial flow within only the viable areas of an infarct and can be used to measure RFR. The present study assessed in patients with chronic myocardial infarction whether microvascular resistance in the infarct is different from that in the reference area. Therefore, the correlation between FFR and RFR using $\mathrm{H}_{2}{ }^{15} \mathrm{O}$ PET was studied. Methods: In the catheterization laboratory, FFR was measured in the infarct-related artery and a reference coronary artery. The $\mathrm{H}_{2}{ }^{15} \mathrm{O}$ PET study and FFR measurements were performed on the same day in 22 patients. Results: In 27 patients, the mean interval between the PET study and infarction was $3.3 \mathrm{y}$. Most patients had an anterior infarction, and the mean ejection fraction was $44 \%$. The mean FFR and RFR values were $0.75 \pm 0.16$ and $0.74 \pm 0.18$, respectively. A significant correlation $(r=0.81 ; P<0.0001)$ was found between FFR and RFR. The linear regression line was close to the line of identity. Conclusion: In patients with chronic myocardial infarction and a reduced ejection fraction, a good correlation was found between FFR measurements in the infarct-related artery and RFR. Because the linear regression line between FFR and RFR was close to the line of identity, one can conclude that microvascular resistance in the viable myocardium does not differ from that in the reference area.

Received Jun. 20, 2007; revision accepted Aug. 27, 2007.

For correspondence or reprints contact: Koen M. Marques, MD, Department of Cardiology, VU University Medical Center, De Boelelaan 1117, P.O. Box 7057, 1007 MB Amsterdam, The Netherlands.

E-mail: km.marques@VUmc.n

COPYRIGHT @ 2007 by the Society of Nuclear Medicine, Inc.
Key Words: fractional flow reserve; relative flow reserve; myocardial infarction

J Nucl Med 2007; 48:1987-1992

DOI: 10.2967/jnumed.107.044370

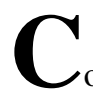

oronary angiography remains one of the cornerstones in the evaluation of patients with coronary artery disease. However, the hemodynamic significance of a stenosis is difficult to assess from the angiogram alone. Measurement of fractional flow reserve (FFR) with a pressure wire is a simple and reliable index to determine the functional significance of a coronary stenosis. In patients with preserved left ventricular function, a well-defined cutoff of 0.75 has been shown to accurately distinguish hemodynamically significant stenoses (1-6).

In patients with a prior myocardial infarction, however, measurement of FFR in the infarct-related artery may be biased, for 2 reasons: the mass of viable myocardium depending on the stenotic infarct-related artery is smaller for a similar degree of stenosis, and microvascular resistance may be greater in the infarcted area than in the reference area, possibly blunting maximal hyperemia. To investigate the value of FFR measurements in patients with prior infarction, one needs to address these 2 potential pitfalls. The first issue can be addressed by using $\mathrm{H}_{2}{ }^{15} \mathrm{O}$ PET to measure myocardial flow. $\mathrm{H}_{2}{ }^{15} \mathrm{O}$ PET provides perfusion data only on the viable myocardium within an infarcted region (7-9). Because flow is expressed per milliliter of perfusable (viable) tissue, the smaller amount of viable myocardium within the infarcted area is accounted for and, thus, comparison of blood flow between the infarcted and reference myocardial areas is possible. The second issue can be examined by correlating FFR with relative flow reserve (RFR). FFR is the ratio of hyperemic myocardial flow in the stenotic area to maximal flow in that same territory without stenosis (4). RFR is the ratio of myocardial blood flow in the stenotic area to blood flow in a normally perfused reference area, at maximal hyperemia 
(10). If microvascular function in the viable, chronically infarcted myocardium does not differ from that in the reference area, and if the reference coronary artery has no stenosis, FFR should equal RFR; if microvascular resistance is higher in the infarcted area than in the reference area, FFR will be higher than RFR.

The present study assessed whether, in patients with chronic infarction, microvascular resistance in the infarcted area is different from that in the reference area. Therefore, the correlation between FFR and RFR measurements was examined using ${ }^{15} \mathrm{O}$-labeled water and PET. Furthermore, the best FFR cutoff was determined in these patients, based on the results of noninvasive stress testing.

\section{MATERIALS AND METHODS}

\section{Study Population}

Patients were included in the study if they fulfilled the following criteria: a documented single myocardial infarction more than 2 mo before the study, a nonoccluded infarct-related artery with a proximal culprit lesion, and at least one normal non-infarct-related coronary artery. Patients whose infarct-related or normal noninfarct-related artery was a nondominant right coronary artery were not included. PET and FFR measurements were performed on the same day in 22 patients; in the remaining 5 patients, the interval between the 2 procedures was less than $1 \mathrm{wk}$. For all patients, recent information was available from dobutamine stress echocardiography or ${ }^{99 \mathrm{~m}} \mathrm{Tc}$-sestamibi perfusion scintigraphy on the extent and location of the infarcted area and whether it was ischemic. The study was approved by the medical ethical committee of the VU University Medical Centre. Written informed consent was obtained from all patients.

\section{Myocardial Perfusion Scintigraphy}

SPECT was performed according to a 2-d stress/rest protocol. Exercise or adenosine was used for the stress images. ${ }^{99 \mathrm{~m}} \mathrm{Tc}$-labeled sestamibi was injected at maximal exercise or after intravenous infusion of adenosine $(0.14 \mathrm{mg} / \mathrm{kg}$ per minute). SPECT was performed using a 2-head $\gamma$-camera equipped with low-energy highresolution collimators. The images were acquired using a $360^{\circ}$ circular orbit and were analyzed using a 13-segment model (11). Stress and rest segments were semiquantitatively scored as normal (grade 0 ) or as having a mild, moderate, or severe (grade 3) perfusion defect. Perfusion defects were allocated to the territory of a coronary artery. Defects in the anterior and anteroseptal region were allocated to the left anterior descending coronary artery, defects in the posterolateral wall to the left circumflex coronary artery, and inferior and basal inferoseptal defects to the right coronary artery. Apical defects were considered to be in the region of the left anterior descending coronary artery, unless the defect extended to the posterolateral (left circumflex coronary artery) or inferior (right coronary artery) wall. Perfusion defects were considered to be reversible and positive for the presence of ischemia when the rest perfusion score improved by one grade or more over the stress score. Segments with irreversible perfusion defects or normal perfusion were considered negative for the presence of ischemia. The scintigrams were scored by 2 experienced cardiologists; in cases of disagreement, a third cardiologist scored the images to achieve a majority decision.

\section{Dobutamine Stress Echocardiography}

An intravenous infusion of dobutamine was started at a rate of 10 $\mu \mathrm{g} / \mathrm{kg}$ per minute and was increased by $10 \mu \mathrm{g} / \mathrm{kg}$ per minute every 3 min until either wall motion abnormalities were observed or a maximal rate of $40 \mu \mathrm{g} / \mathrm{kg}$ per minute was reached. In patients who did not reach $90 \%$ of their age-adjusted maximal heart rate and who had no objective signs of ischemia, $0.25 \mathrm{mg}$ of atropine was given every minute up to a maximum of $1.0 \mathrm{mg}$ while the dobutamine infusion was continued. Endpoints for stopping the infusion were the same as mentioned in the guidelines (12). Two-dimensional echocardiography was performed, obtaining parasternal long- and short-axis views and apical 4- and 2-chamber views. Imaging was performed throughout the study and during recovery until resolution of new wall motion abnormalities. Online digital images in quad screen format were analyzed for the presence, extent, severity, and location of segmental wall motion abnormalities. Myocardial contractile function was graded as normal, hypokinetic, akinetic, or dyskinetic in each segment. An echocardiographic stress test was considered positive when new or worsening stress-induced wall motion abnormalities were observed. The standard algorithm was used to assign ventricular segments to coronary territories: left anterior descending coronary artery (basal and midanteroseptal segments; basal, mid, and apical anterior segments; and mid and apical septal and apical lateral segments), left circumflex coronary artery (basal and mid lateral segments and basal and mid posterior segments), and right coronary artery (basal, mid-, and apical inferior segments and basal septal segments) (13). The dobutamine stress echocardiograms were scored by 2 experienced cardiologists; in cases of disagreement, a third cardiologist scored the images to achieve a majority decision.

\section{PET Study}

A short rectilinear transmission scan was performed to ascertain that the heart was positioned in the center of the view of the scanner. Then, a complete transmission scan was obtained to correct the subsequent emission scans for tissue attenuation. After the transmission scan, myocardial perfusion was measured using a dynamic $\mathrm{H}_{2}{ }^{15} \mathrm{O}$ scan sequence. This measurement was taken at baseline and after hyperemia, induced by intravenous administration of adenosine $(140 \mu \mathrm{g} / \mathrm{kg} / \mathrm{min})$. Finally, a static $\mathrm{C}^{15} \mathrm{O}$ scan was obtained to image the blood volume. Anatomic tissue images were generated by subtracting the blood pool from the transmission images. Transaxial anatomic tissue images of the left ventricle were reoriented according to the anatomic axis of the heart and displayed as short-axis slices. The same reslicing parameters were applied to the dynamic $\mathrm{H}_{2}{ }^{15} \mathrm{O}$ images. Regions of interest were defined manually on the transaxial images at the basal, midventricular, and apical levels. Each basal and midventricular slice was divided in 6 equidistant sectors starting from the posterior insertion of the right ventricular free wall into the left ventricle. Corresponding regions of interest from a variable number of slices were grouped in each patient to generate 13 volumes of interest ( 6 basal, 6 midventricular, and 1 apical). Additional regions of interest were defined in the left and right ventricular chambers. The latter set was projected on the dynamic $\mathrm{H}_{2}{ }^{15} \mathrm{O}$ images to generate image-derived input functions. The standard single-tissue-compartment model was used together with these input functions to determine myocardial blood flow (mL/ $\mathrm{min} / \mathrm{mL}$ of perfusable tissue), including intrinsic corrections for spillover from both left and right ventricles (14).

The original 13 regions of interest were regrouped into 5 larger segments (anteroseptum, inferoseptum, anterior, lateral, and 
inferoposterior walls). The apex and the anterior and anteroseptal walls were allocated to the left anterior descending coronary artery, the lateral wall to the left circumflex coronary artery, and the inferoseptum and inferoposterior wall to the right coronary artery. The RFR was defined as the ratio of myocardial perfusion in the infarcted area to perfusion in the contralateral normally perfused area, at hyperemia.

\section{Quantitative Angiography and FFR Measurement}

A 6- or 7-French guiding catheter was positioned in the coronary ostium, and the aortic pressure was measured. After intracoronary administration of $0.2 \mathrm{mg}$ of isosorbide dinitrate, an angiogram of the infarct-related artery was made in 2 orthogonal projections. Nitrates were given to maximize epicardial dilatation. In this way, flow-mediated vasodilatation, which may affect the FFR measurement, was prevented. In addition, nitrates prevent coronary spasm from being induced by the presence of the wire in the coronary stenosis. Quantitative coronary angiography was performed offline using the CAAS II system (Pie Medical Data). A pressure wire (WaveWire; Volcano Therapeutics) was advanced to the tip of the guiding catheter. At that time, both pressures were verified to be identical. Then, the wire was positioned distal to the coronary stenosis and the distal pressure was recorded. Maximal hyperemia was induced by intracoronary administration of $40 \mu \mathrm{g}$ of adenosine. FFR was calculated as the ratio of distal pressure to aortic pressure at maximal hyperemia. Afterward, FFR was also determined in the contralateral reference coronary artery.

\section{Statistics}

All data are presented as mean \pm SD. Differences in continuous variables between groups were compared using the Student $t$ test. The relationship between FFR and RFR was assessed using linear regression analysis. A value of $P$ less than 0.05 was considered significant. Receiver operator characteristics were calculated to determine the best FFR cutoff value to predict ischemia in the infarcted area by noninvasive testing. The sensitivity, specificity, and FFR cutoff to determine ischemia were calculated.

\section{RESULTS}

The clinical characteristics of the 27 patients are summarized in Table 1. The interval between the PET study and myocardial infarction varied widely. Most infarctions were at the anterior wall. The infarction was treated by primary coronary angioplasty in 8 patients, thrombolysis in 11 patients, and rescue angioplasty in 2 patients. In 6 patients, no reperfusion therapy was performed. The mean ejection fraction was $44 \% \pm 15 \%$.

Table 2 displays the angiographic, hemodynamic, and perfusion data. The percentage diameter stenosis in the infarct-related artery ranged from $0 \%$ to $90 \%$. FFR was significantly lower in the infarct-related artery than in the reference artery. In the infarcted region, FFR ranged from 0.34 to 1.0 (mean, $0.75 \pm 0.16$ ) and RFR from 0.24 to 1.06 (mean, $0.74 \pm 0.18$ ). Myocardial blood flow in the infarcted region was significantly lower at baseline and during hyperemia. The hyperemic rate-pressure products during catheterization and during the PET study were not statistically different. A good correlation was found between FFR and RFR (Fig. 1A). The mean difference between RFR and FFR was
TABLE 1

Clinical Characteristics of Patients $(n=27)$

\begin{tabular}{lc}
\hline \multicolumn{1}{c}{ Parameter } & Value \\
\hline Age $(y)$ & $56 \pm 9$ \\
Male $(n)$ & $23(85 \%)$ \\
STEMI $(n)$ & $22(81 \%)$ \\
Coronary risk factors $(n)$ & \\
Diabetes mellitus & $2(7 \%)$ \\
Hypertension & $9(33 \%)$ \\
Hypercholesterolemia & $17(63 \%)$ \\
Smoking & $15(56 \%)$ \\
Mean interval $( \pm$ SD) between MI and PET $(y)$ & $3.3 \pm 4.4$ \\
Infarct-related artery $(n)$ & \\
LAD & $17(63 \%)$ \\
LCx & $3(11 \%)$ \\
RCA & $7(26 \%)$ \\
Reference coronary artery $(n)$ & \\
LAD & $4(15 \%)$ \\
LCx & $21(78 \%)$ \\
RCA & $2(7 \%)$ \\
Left ventricular ejection fraction $(\%)$ & $44 \pm 15$
\end{tabular}

STEMI = ST-segment elevation myocardial infarction; $\mathrm{Ml}=$ myocardial infarction; LAD = left anterior descending artery; LCx = left circumflex artery; RCA = right coronary artery.

$0.01 \pm 0.11$ (Fig. 1B). To detect ischemia, we performed nuclear scintigraphy on 18 patients and dobutamine stress echocardiography on 9 patients. In 16 patients, the stress test findings were abnormal in the infarcted area. The optimal cutoff of FFR for discriminating reversible ischemia on noninvasive imaging was 0.79 , resulting in a sensitivity of $81 \%$ and a specificity of $82 \%$ (Fig. 2). An FFR cutoff of 0.75 had a sensitivity of $69 \%$ and a specificity of $82 \%$.

\section{DISCUSSION}

In patients with chronic myocardial infarction and a reduced ejection fraction, a good correlation was found

\section{TABLE 2}

Angiographic, Coronary Hemodynamic, and PET Data

\begin{tabular}{|c|c|}
\hline Parameter & Value \\
\hline DS infarct-related artery (\%) & $54 \pm 25$ \\
\hline FFR infarct-related artery & $0.75 \pm 0.16$ \\
\hline FFR reference coronary artery & $0.97 \pm 0.02^{*}$ \\
\hline hRPP during angiography (bpm.mm Hg) & $7,849 \pm 1,587$ \\
\hline \multicolumn{2}{|l|}{ MBF infarcted area $(\mathrm{mL} / \mathrm{min} / \mathrm{mL})$} \\
\hline At baseline & $0.73 \pm 0.20$ \\
\hline At hyperemia & $2.21 \pm 1.02$ \\
\hline \multicolumn{2}{|l|}{ MBF reference area $(\mathrm{mL} / \mathrm{min} / \mathrm{mL})$} \\
\hline At baseline & $0.89 \pm 0.22^{*}$ \\
\hline At hyperemia & $2.92 \pm 1.11^{*}$ \\
\hline RFR & $0.74 \pm 0.18$ \\
\hline hRPP during PET study (bpm.mm Hg) & $8,479 \pm 1,805$ \\
\hline \multicolumn{2}{|c|}{$\begin{array}{l}{ }^{*} P<0.0001 \text { for reference vs. infarcted area. } \\
\text { DS }=\text { diameter stenosis; hRPP = hyperemic rate-pressure } \\
\text { roduct; MBF = myocardial blood flow. }\end{array}$} \\
\hline
\end{tabular}



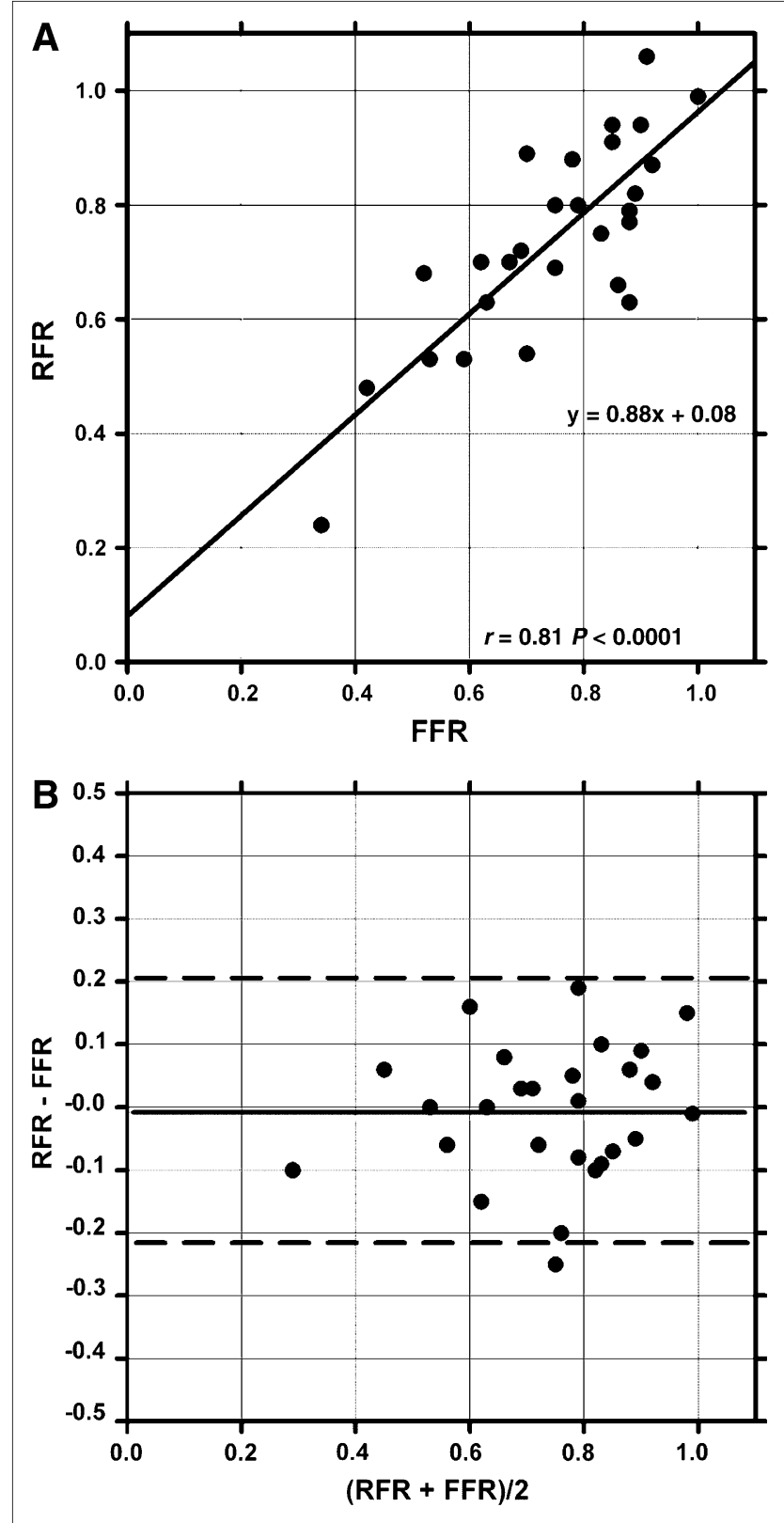

FIGURE 1. (A) Plot of relationship between RFR as assessed by PET and RFR as assessed by FFR. (B) Bland-Altman plot of FFR and RFR. Solid line represents mean difference; dashed lines represent 2 SDs from this mean.

between FFR measurements in the infarct-related artery and RFR, determined with $\mathrm{H}_{2}{ }^{15} \mathrm{O}$ PET. The linear regression line between FFR and RFR was close to the line of identity. This finding implies that in patients with chronic myocardial infarction, microvascular resistance does not differ between the infarcted and reference areas.

\section{Rationale for Measuring RFR with $\mathrm{H}_{2}{ }^{15} \mathrm{O}$ PET in Chronic Myocardial Infarction}

$\mathrm{H}_{2}{ }^{15} \mathrm{O}$ PET provides quantitative information on regional flow in the myocardium $(15,16)$. In patients with chronic infarction, the amount of viable myocardium is decreased

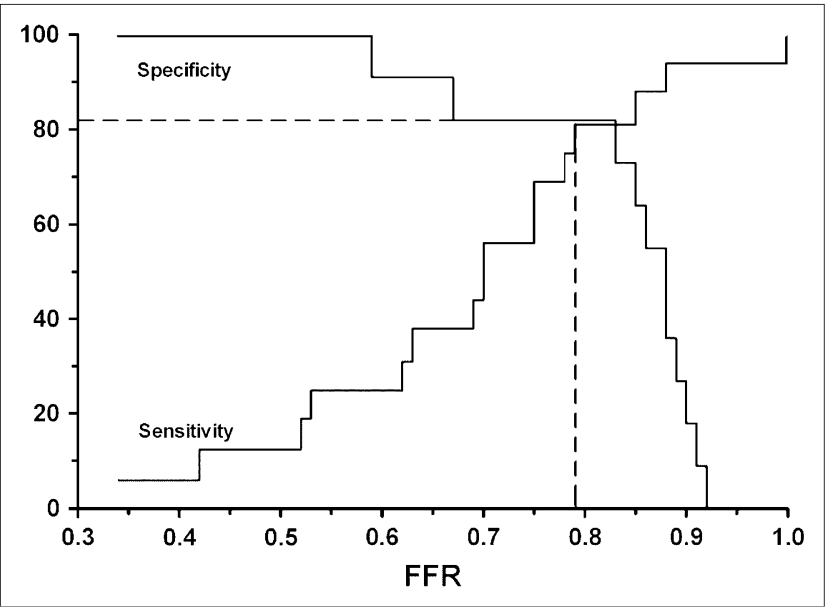

FIGURE 2. Sensitivity and specificity curves for ability of FFR to detect ischemia by noninvasive stress testing.

and so also is maximal achievable blood flow to the infarcted area, even in the absence of an infarct-related artery stenosis. Because the $\mathrm{H}_{2}{ }^{15} \mathrm{O}$ PET technique expresses flow per milliliter of perfusable (viable) tissue only (and not per milliliter of total tissue in the infarcted area), the decreased amount of viable myocardium within the infarcted area is accounted for and, thus, comparison of blood flow between infarcted and reference myocardial areas is possible.

\section{Correlation Between FFR and RFR in Chronic Myocardial Infarction}

In patients without prior myocardial infarction, FFR measurements have been validated through comparison with RFR (17). FFR equals RFR if 3 conditions are fulfilled. The first of these is that significant narrowing of the reference coronary artery be absent; therefore, in the present study, FFR in the reference coronary artery was measured. The second condition is that FFR and RFR measurements be performed under similar hemodynamic conditions; in the present study, the rate-pressure products at maximal hyperemia during FFR measurement and during PET did not differ significantly. The third condition is that microvascular resistance be identical in the stenotic and contralateral areas during maximal hyperemia. This condition does not mean that microvascular resistance had to be normal in the reference and infarcted areas. Coronary flow reserve and maximal myocardial blood flow have been shown to be significantly higher in healthy volunteers than in myocardial regions perfused by normal coronary arteries in patients with remote areas of myocardial ischemia (18). In concordance with the data of Uren et al., maximal myocardial blood flow data measured in volunteers at our institution $(4.31 \pm 0.90 \mathrm{~mL} / \mathrm{min} / \mathrm{mL})$ were also significantly higher than the reference area flow data in the present study (19). The implication is that microvascular resistance in the reference area in the present study was higher than truly normal resistance. However, maximal myocardial blood flow data and absolute flow reserve in the reference area $(3.3 \pm$ 1.2) were comparable between the present study and other 
$\mathrm{H}_{2}{ }^{15} \mathrm{O}$ PET studies on patients with coronary artery disease $(18,20-22)$. This comparability suggests that the degree of microvascular functional impairment in the present study was similar to that found by other studies.

In the acute and subacute phases of a myocardial infarction, microvascular resistance in the infarcted area can be increased (23-25). There are no data on microcirculatory resistance in chronically infarcted areas in patients. If microcirculatory function in a chronically infarcted area is similar to that in a reference area, FFR in an infarct-related artery should be identical to RFR. If microvascular resistance in a chronically infarcted area remains greater than that in a reference area, FFR will be higher than RFR. In this situation, FFR will overestimate RFR proportionally by the degree to which microvascular resistance is higher in the infarcted than the reference area. In the present study, RFR was measured with $\mathrm{H}_{2}{ }^{15} \mathrm{O}$ PET. In infarcted areas, $\mathrm{H}_{2}{ }^{15} \mathrm{O}$-PET provides perfusion data only on tissue that is capable of exchanging water rapidly-a property of viable myocardium (7-9). Myocardial blood flow in nonperfusable (scar) tissue is negligible. The fact that in the present study the regression line of the relationship between FFR and RFR approached the line of identity (Fig. 1A), and that the mean difference in the Bland-Altman plot was not significantly different from zero (Fig. 1B), indicates that microvascular resistance did not significantly differ between the infarcted and reference areas.

\section{The FFR Cutoff After Myocardial Infarction}

In the present study, the optimal cutoff of FFR to detect ischemia in the infarcted area was 0.79 , resulting in a sensitivity of $81 \%$ and a specificity of $82 \%$. An FFR cutoff of 0.75 had a sensitivity of $69 \%$ and a specificity of $82 \%$. These findings are in line with those of previous studies, performed during the subacute phase of an infarction. De Bruyne et al. found in 57 patients with a recent myocardial infarction (average time, $20 \mathrm{~d}$ ) that the best cutoff of FFR was 0.78 (26). In another study, FFR measurements were compared with SPECT $4 \mathrm{~d}$ after myocardial infarction. The optimal FFR for discriminating inducible ischemia also was 0.78 (27). Usui et al. compared the reliability of FFR in infarct-related and non-infarct-related artery stenoses. They found a similar sensitivity and specificity in patients with and without prior infarction at an FFR cutoff of 0.75 (28). In all 3 studies, the ejection fraction was rather well preserved $(58 \%, 53 \%$, and $56 \%$, respectively). The present study extends the validity of the 0.75 FFR cutoff to patients with chronic infarction and considerably diminished left ventricular function.

\section{Limitations}

PET and pressure measurements were not performed simultaneously. However, no difference in rate-pressure products between the 2 procedures was observed at hyperemia. Furthermore, hyperemia during PET and pressure measurements was induced by intravenous and intracoronary adenosine administration, respectively. Studies have shown, however, that both intracoronary and intravenous adminis- tration of adenosine can achieve maximal hyperemia (29). In the postinfarction setting, the accuracy of noninvasive stress testing is decreased, possibly influencing the FFR cutoff and its sensitivity and specificity. The FFR cutoff in the chronic phase of myocardial infarction should be confirmed in a larger study population.

\section{CONCLUSION}

The present study showed that in patients with chronic myocardial infarction, FFR correlated well with RFR, as assessed by $\mathrm{H}_{2}{ }^{15} \mathrm{O}$ PET. The implication is that in patients with chronic myocardial infarction, microvascular resistance in viable myocardium did not differ from that in the reference area. Because microvascular resistance was similar to that found by previous studies on patients, the established 0.75 FFR threshold also is applicable to patients with chronic infarction. In many patients with coronary artery disease, including the present study population, microvascular function is worse than in healthy persons.

\section{ACKNOWLEDGMENT}

This study was supported by grant 98.102 from the Netherlands Heart Foundation.

\section{REFERENCES}

1. Pijls NH, de Bruyne B, Peels K, et al. Measurement of fractional flow reserve to assess the functional severity of coronary-artery stenoses. N Engl J Med. 1996; 334:1703-1708.

2. Pijls NH, van Son JA, Kirkeeide RL, de Bruyne B, Gould KL. Experimental basis of determining maximum coronary, myocardial, and collateral blood flow by pressure measurements for assessing functional stenosis severity before and after percutaneous transluminal coronary angioplasty. Circulation. 1993;87: 1354-1367.

3. de Bruyne B, Bartunek J, Sys SU, Heyndrickx GR. Relation between myocardial fractional flow reserve calculated from coronary pressure measurements and exercise-induced myocardial ischemia. Circulation. 1995;92:39-46.

4. Pijls NH, Van Gelder B, Van Der Voort PH, et al. Fractional flow reserve: a useful index to evaluate the influence of an epicardial coronary stenosis on myocardial blood flow. Circulation. 1995;92:3183-3193.

5. Bartunek J, Van Schuerbeeck E, de Bruyne B. Comparison of exercise electrocardiography and dobutamine echocardiography with invasively assessed myocardial fractional flow reserve in evaluation of severity of coronary arterial narrowing. Am J Cardiol. 1997;79:478-481.

6. Fearon WF, Takagi A, Jeremias A, et al. Use of fractional myocardial flow reserve to assess the functional significance of intermediate coronary stenoses. Am J Cardiol. 2000;86:1013-1014.

7. Yamamoto Y, de Silva R, Rhodes CG, Araujo L, Iida H. A new strategy for the assessment of viable myocardium and regional myocardial blood flow using 15O-water and dynamic positron emission tomography. Circulation. 1992;86: 167-178.

8. Bol A, Melin JA, Vanoverschelde JL, et al. Direct comparison of $\left[{ }^{13} \mathrm{~N}\right]$ ammonia and $\left[{ }^{15} \mathrm{O}\right]$ water estimates of perfusion with quantification of regional myocardial blood flow by microspheres. Circulation. 1993;87:512-525.

9. Iida H, Tamura Y, Kitamura K, Bloomfield PM, Eberl S, Ono Y. Histochemical correlates of ${ }^{15} \mathrm{O}$-water-perfusable tissue fraction in experimental canine studies of old myocardial infarction. J Nucl Med. 2000;41:1737-1745.

10. Gould KL, Kirkeeide RL, Buchi M. Coronary flow reserve as a physiologic measure of stenosis severity. J Am Coll Cardiol. 1990;15:459-474.

11. Ritchie J, Bateman TM, Bonow RO, et al. Guidelines for clinical use of cardiac radionuclide imaging: a report of the American Heart Association/American College of Cardiology Task Force on Assessment of Diagnostic and Therapeutic Cardiovascular Procedures, Committee on Radionuclide Imaging, developed in 
collaboration with the American Society of Nuclear Cardiology. Circulation. 1995;91:1278-1303.

12. Quinones MA, Douglas PS, Foster E, et al. American College of Cardiology/ American Heart Association clinical competence statement on echocardiography: a report of the American College of Cardiology/American Heart Association/ American College of Physicians-American Society of Internal Medicine Task Force on Clinical Competence. Circulation. 2003;107:1068-1089.

13. Feigenbaum $\mathrm{H}$. Echocardiography and coronary artery disease. Int $J$ Card Imaging. 1993;9(suppl 2):55-67.

14. Hermansen F, Rosen SD, Fath-Ordoubadi F, et al. Measurement of myocardial blood flow with oxygen-15 labelled water: comparison of different administration protocols. Eur J Nucl Med. 1998;25:751-759.

15. Araujo LI, Lammertsma AA, Rhodes CG, et al. Noninvasive quantification of regional myocardial blood flow in coronary artery disease with oxygen-15labeled carbon dioxide inhalation and positron emission tomography. Circulation. 1991;83:875-885.

16. Iida H, Kanno I, Takahashi A, et al. Measurement of absolute myocardial blood flow with $\mathrm{H}_{2}{ }^{15} \mathrm{O}$ and dynamic positron-emission tomography: strategy for quantification in relation to the partial-volume effect. Circulation. 1988;78:104-115.

17. de Bruyne B, Baudhuin T, Melin JA, et al. Coronary flow reserve calculated from pressure measurements in humans: validation with positron emission tomography. Circulation. 1994;89:1013-1022.

18. Uren NG, Marraccini P, Gistri R, de Silva R, Camici PG. Altered coronary vasodilator reserve and metabolism in myocardium subtended by normal arteries in patients with coronary artery disease. J Am Coll Cardiol. 1993;22:650-658.

19. Dijkmans PA, Knaapen P, Sieswerda GT, et al. Quantification of myocardial perfusion using intravenous myocardial contrast echocardiography in healthy volunteers: comparison with positron emission tomography. J Am Soc Echocardiogr. 2006;19:285-293.
20. Yoshinaga K, Katoh C, Noriyasu K, et al. Reduction of coronary flow reserve in areas with and without ischemia on stress perfusion imaging in patients with coronary artery disease: a study using oxygen 15-labeled water PET. J Nucl Cardiol. 2003;10;275-283.

21. Uren NG, Melin JA, de Bruyne B, Wijns W, Baudhuin T, Camici PG. Relation between myocardial blood flow and the severity of coronary-artery stenosis. NEngl J Med. 1994;330:1782-1788.

22. Miller DD, Donohue TJ, Wolford TL, Kern MJ, Bergmann SR. Assessment of blood flow distal to coronary artery stenoses: correlations between myocardial positron emission tomography and poststenotic intracoronary Doppler flow reserve. Circulation. 1996;94:2447-2454.

23. Agati L. Microvascular integrity after reperfusion therapy. Am Heart J. 1999; 138(suppl):S76-S78.

24. Ito H, Tomooka T, Sakai N, et al. Lack of myocardial perfusion immediately after successful thrombolysis: a predictor of poor recovery of left ventricular function in anterior myocardial infarction. Circulation. 1992;85:1699-1705.

25. Reffelmann T, Kloner RA. The "no-reflow" phenomenon: basic science and clinical correlates. Heart. 2002;87:162-168.

26. de Bruyne B, Pijls NH, Bartunek J, et al. Fractional flow reserve in patients with prior myocardial infarction. Circulation. 2001;104:157-162.

27. Samady H, Lepper W, Powers ER, et al. Fractional flow reserve of infarct-related arteries identifies reversible defects on noninvasive myocardial perfusion imaging early after myocardial infarction. J Am Coll Cardiol. 2006;47:2187-2193.

28. Usui Y, Chikamori T, Yanagisawa H, et al. Reliability of pressure-derived myocardial fractional flow reserve in assessing coronary artery stenosis in patients with previous myocardial infarction. Am J Cardiol. 2003;92:699-702.

29. de Bruyne B, Pijls NH, Barbato E, et al. Intracoronary and intravenous adenosine 5 '-triphosphate, adenosine, papaverine, and contrast medium to assess fractional flow reserve in humans. Circulation. 2003;107:1877-1883. 